\title{
The Simulation of Conveyor Control System Using the Virtual Commissioning and Virtual Reality
}

\author{
Roman Ružarovský ${ }^{*}$, Radovan Holubek', Daynier Rolando Delgado Sobrino', \\ Matej Janíček ${ }^{1}$ \\ 1 Institute of Production Technologies, Faculty of Materials Science and Technology in Trnava, Slovak University \\ of Technology in Bratislava, Jána Bottu 2781/25, 91724 Trnava, Slovakia \\ * Corresponding author's e-mail: roman.ruzarovsky@stuba.sk
}

\begin{abstract}
Designing of the conveyors and its control system with control program through the design tool "Virtual Commissioning" is very important in the digital era Industry 4.0. Virtual Commissioning was recently used to perform realistic virtual simulations in the early stages of development processes in automation of the conveyors too. The main benefit is the possibility of integrating and testing the control system through the simulation on the virtual model even before physically constructing the conveyor. The contribution focuses on the possibilities of transforming a real conveyor into a virtual model in the Siemens Tecnomatix Process Simulate program and integrating the Siemens S7-300 real control system to control the conveyor itself. In the model it was allowed to test and perform numerous variations and situations without physical intervention into the real conveyor and possible damage functional parts including through virtual reality. These experiments were subsequently evaluated and the control system optimized with respect to material flow and the developed PLC code on virtual model was verified on real conveyor control system.
\end{abstract}

Keywords: simulation, conveyor control, sensor integration, virtual commissioning, virtual reality.

\section{INTRODUCTION}

Designing of the conveyors as technological devices is a comprehensive set of tasks to be solved in basic sequential steps. Currently, various design tools with computer support are used to design a physical model (CAD) according to predefined requirements. the physical model is static without any possibility of verifying its functional model as a moving mechatronic unit. The conveyor as a mechatronic device consists of a physical mechanical model, a motion device (motor + gearbox) and a controller that controls the movement of the motor and the conveyor itself. The development of the control system is carried out during the design of the conveyor's physical model itself, but it is generally tested on a real physical conveyor and assembled conveyer, so putting it into operation is solved directly on the conveyor.
The virtual commissioning as the simulation tool allows you to verify the control program even earlier before the real commissioning as discussed elsewhere $[1,2]$. This tool is an experimental method where the real conveyor is replaced by a computer model. Conveyor units represent a very important part within majority of logistic systems. Their role is to transport variety of materials and foster the functioning of individual technological processes [3]. On the conveyor model, it is possible to test and perform a large number of variations and situations without physical intervention to the actual conveyor and possible damage to the functional parts, as Reinhart said [4]. Reliable, trouble-free operation of continuous transport systems requires regular monitoring and evaluation of each operational indicator [5]. These experiments can then be evaluated and the control system optimized, e.g. with respect to the material flow [6], the times and overall energy consump- 


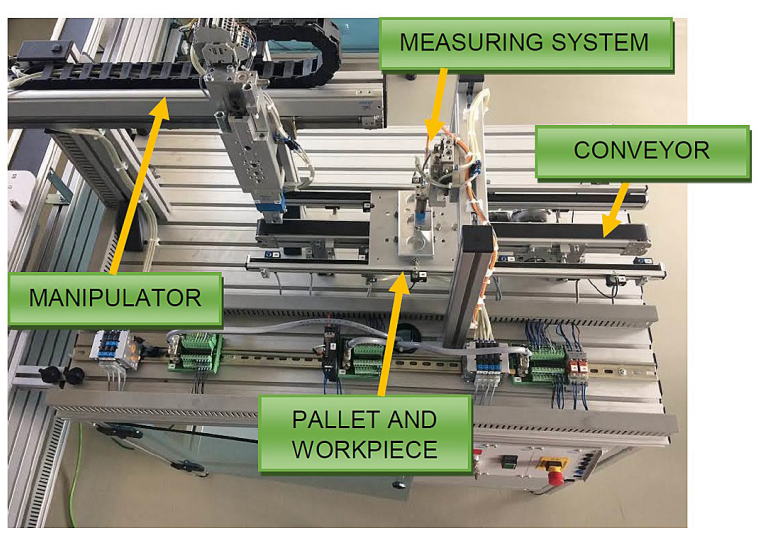

Fig. 1. Automated Quality-handling station with conveyor in iCIM flexible manufacturing system

tion by Zhang [7] during transporting the material. Simulation is a method that allows you to experiment with the conveyor model before it was physically in operation [8]. First and foremost is to create a model for simulation. The model is a simplified representation of the conveyor system used of the modulated elements, rules and features, whose layout, interconnection, but mainly functioning, corresponds to the real system. So the model is quite similar to the real system we are looking at from a certain point of view.

\section{ANALYSIS OF THE PROBLEMATICS AND OBJECTIVES}

The aim of the research is to create an experimental model to explore the possibility of implementing a conveyor control system. This model will be based on a virtual simulation model of the conveyor.

\section{Case study}

The case study is a conveyor integrated in an automated control station. This station is part of the flexible manufacturing system iCIM 3000 from Festo Didactic GmBH. This system is located in the Intelligent Manufacturing Systems Laboratory at the Institute of Production Technologies, MTF STU [9], you can see in paper by Holubek et al.. This station consists of several devices, air 3-axes Cartesian type manipulator with a pneumatic gripper, a measuring device for measuring the size of drill holes and a belt conveyor for conveying the pallets with products "Baseplate", Figure 1.

\section{Belt conveyor and its control system}

The basic concept of the conveyor is a belt driven by a $36 \mathrm{~V}$ DC DC motor with a gearbox from Dunkermotoren $\mathrm{GmBH}$. The conveyor is $700 \mathrm{~mm}$ in length, Figure 2.

The conveyor is designed to transport the "Baseplate" workpieces stored on the system pallet. Through the DC motor, it is possible to transport one palette from the manipulator to the measuring system at the end of the conveyor or in the opposite direction. The pallet can be stopped in 4 positions at the end of both sides of the conveyor and at the measuring point in both directions. he position is indexed by an automatic pneumatic stop mechanism and controlled by the Festo induction sensor SIEN-M8. The presence of the workpiece is detected through the Festo optical gate. The speed of the conveyor is determined by the speed of the DC motor and the gearbox and is unchanged.

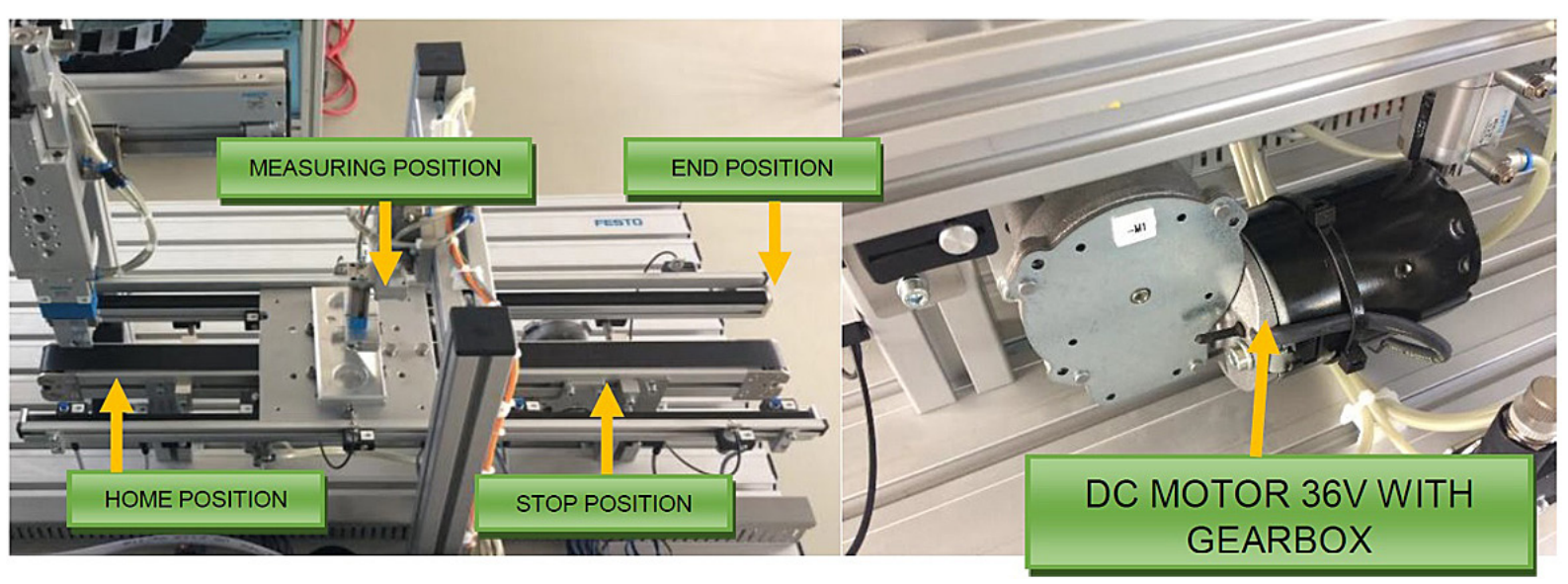

Fig. 2. Belt conveyor with pallet in the measuring position (L) and DC motor with gearbox (R) 


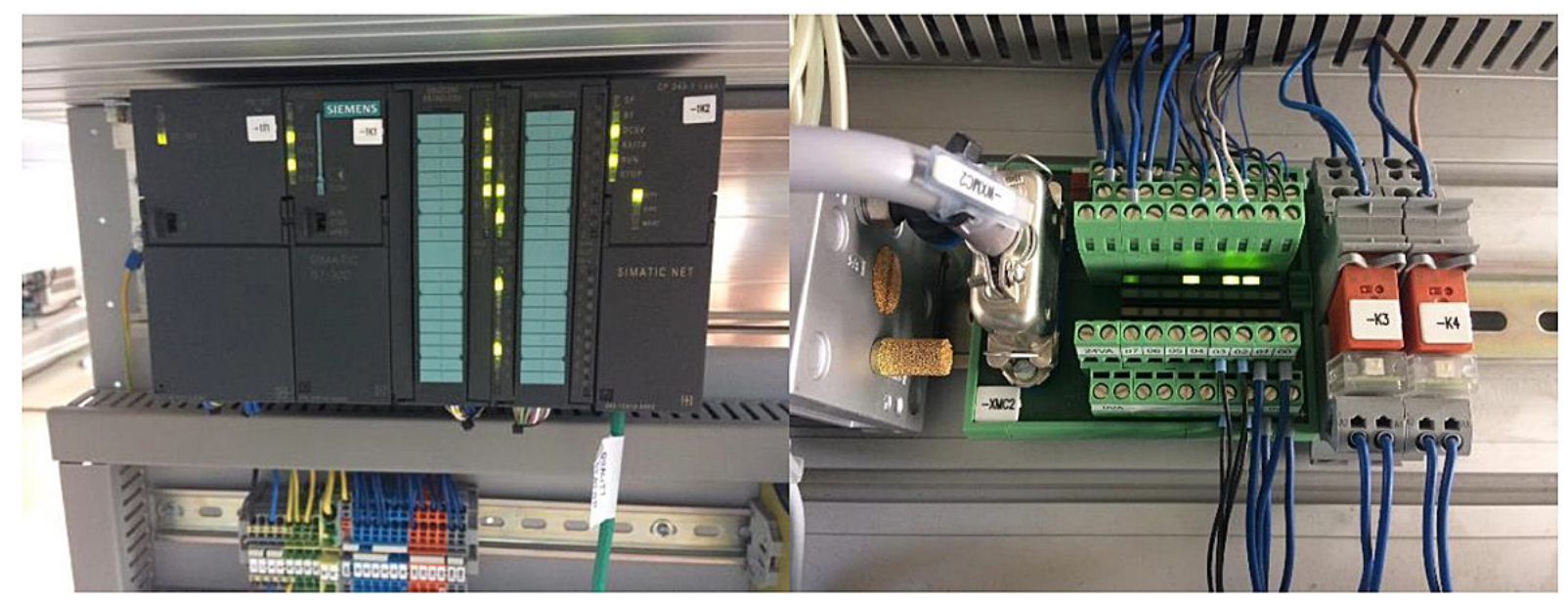

Fig. 3. Control system PLC S7-300 (L) and the electrical terminal block for the conveyor (R)

\section{Objectives}

The requirement for conveyor control is the integration of automation into a real control system. It is used PLC S7-300 control system from Siemens, which controls the manipulator itself and the operation of the measuring device. Specifically, the CPU 313C with the integrated power supply and the CP343-1 communication module on the ProfiNet communication protocol, Figure 3.

It is necessary to integrate the conveyor movement itself, the pallet position sensing and the presence of the workpiece on the pallet. It is also necessary to integrate the HMI for the manual operation of the conveyor itself. The Virtual Commissioning tool is used for testing of the various scenarios without the need for a physical device in the control program developing [10]. Experiments will be evaluated and the control program optimized with respect to the required material flow. Testing the virtual conveyor model for better realistic viewing will be performed through virtual reality.

\section{RESULTS}

In order to be able to test and validate the control program on a virtual model, software that allows it to be used. As a simulation software, we chose Tecnomatix Process Simulate, which allows you to create discrete time and/or event simulations for automated devices and industrial robots. He can communicate with control systems, for example, through the OPC Server, as is described in the Work Flow by Popovič et al. [11]. Virtual Commissioning - need to create
OPC connection - connection between PLC and Process Simulate. The simulator is equipped with a variety of tools to verify the process, including motion, execution, cycle time, and collision detection. The simulation project must include the following phases; i: System analysis and problem definition, formulation of simulation objectives, ii: Collection and processing of process information, estimation of parameters and types, iii: Creating an abstract logic model, iv: Building a model on a computer, $v$ : Verifying and testing the model, vi: Planning and preparation of simulation experiments, viii: Implementation of simulation experiments (model factor changes) or model modification, viii: Evaluation and processing of experimental results.

\section{Virtual Model of the Conveyor}

First of all, it is necessary to create a realistic virtual model, which means that the physical model should be as close as possible to the actual conveyor and, above all, the control have to be realistic. The conveyor model and its surroundings were modelled in the CATIA V5-6R2013 CAD system. The simulation program works with *. jt CAD data, so it was necessary to convert this model to the specified format. JT (Open CAD file) is a $3 \mathrm{D}$ model format developed by Siemens PLM Software. JT files may contain approximate (faceted) data, exact boundary representation surfaces (NURBS), product and manufacturing information (PMI) and metadata. JT files can be exported by native CAD systems and imported by product data management (PDM) systems. Although the JT format is 


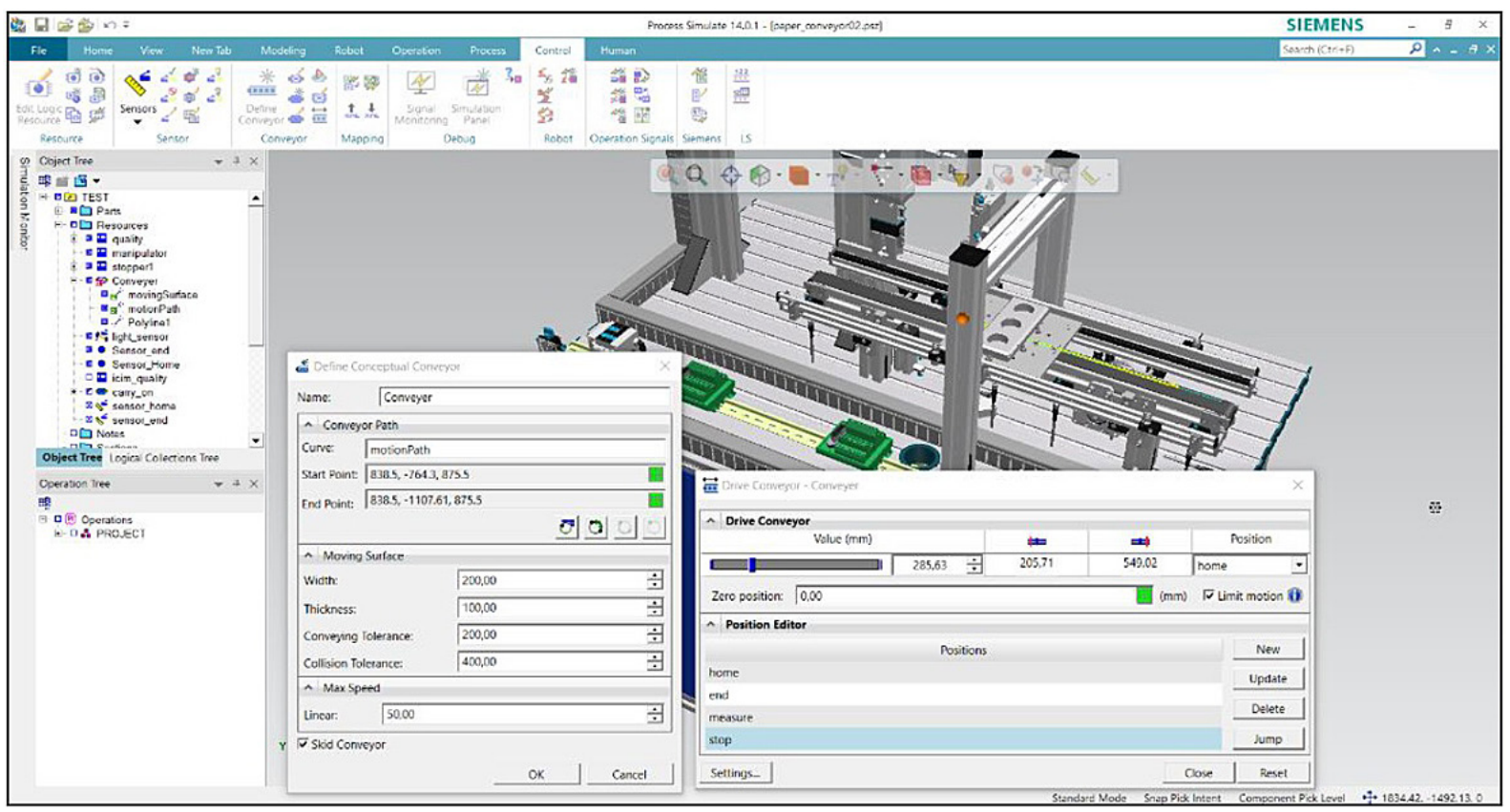

Fig. 4. Definition of the kinematics parameters of the simulation model for the conveyor

primarily used in the automobile and aerospace industries, it can be used for any similar manufacturing industry application [12].

\section{Integration of the model into simulation}

This virtual model has to be integrated into the simulation. Firstly, it is necessary to create a simulation model according to the model of the real system [13]. This means that it is necessary to create the kinematics of the model itself [14] and to define the conditions in which it will move, how much the trajectory will, how the limits and the dynamic parameters (load, speed, acceleration) will be defined. On this system, we can perform simulation experiments at a later stage. When defining a conveyor, it is necessary to define a curve that will move the palette (motionPath), start point, end point, Moving Surface, Max. Speed, definition of Skid, Figure 4.

After defining the parameters, it is possible to design the positions in which the palette can be stopped (Pose Editor) in the simulation model and to manually test the movement itself via the Drive Conveyor command, where the real value of the position is displayed in $\mathrm{mm}$. A virtual model can

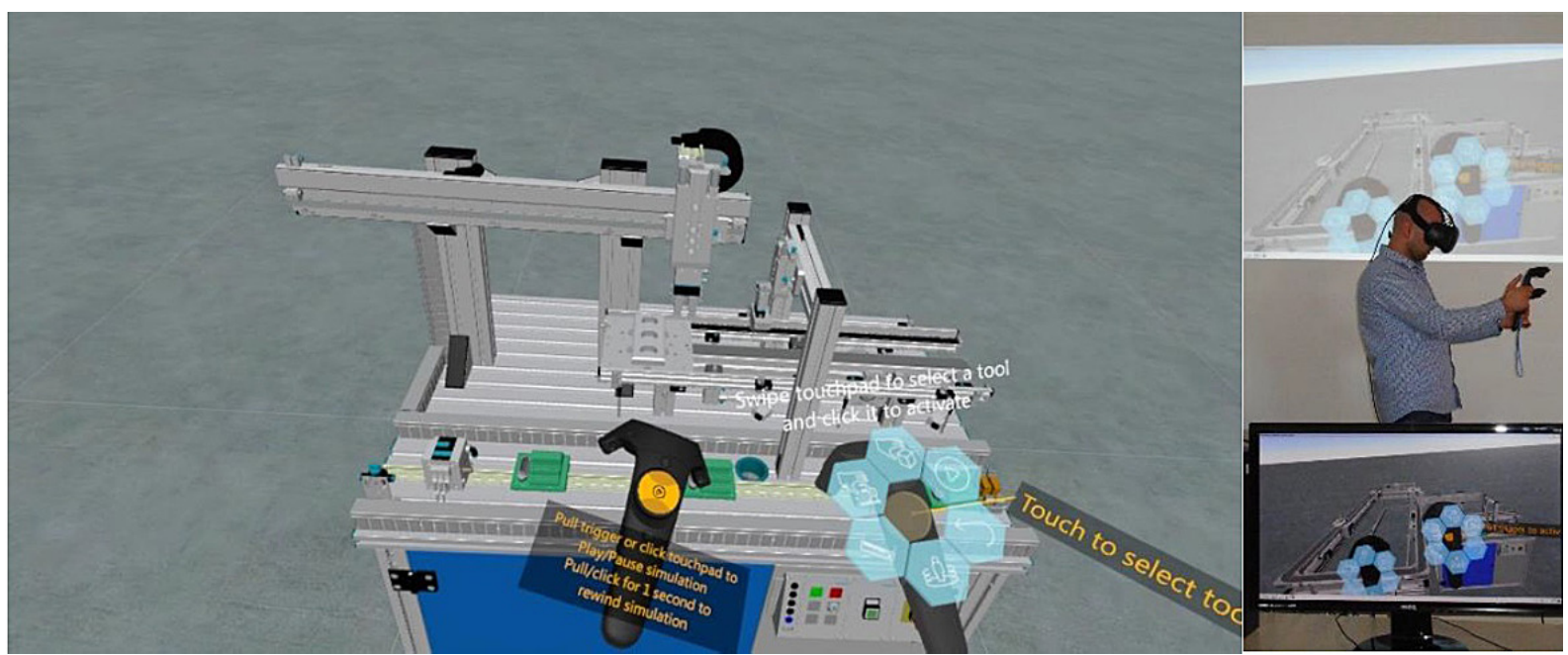

Fig. 5. Definition of the kinematics parameters of the simulation model for the conveyor 


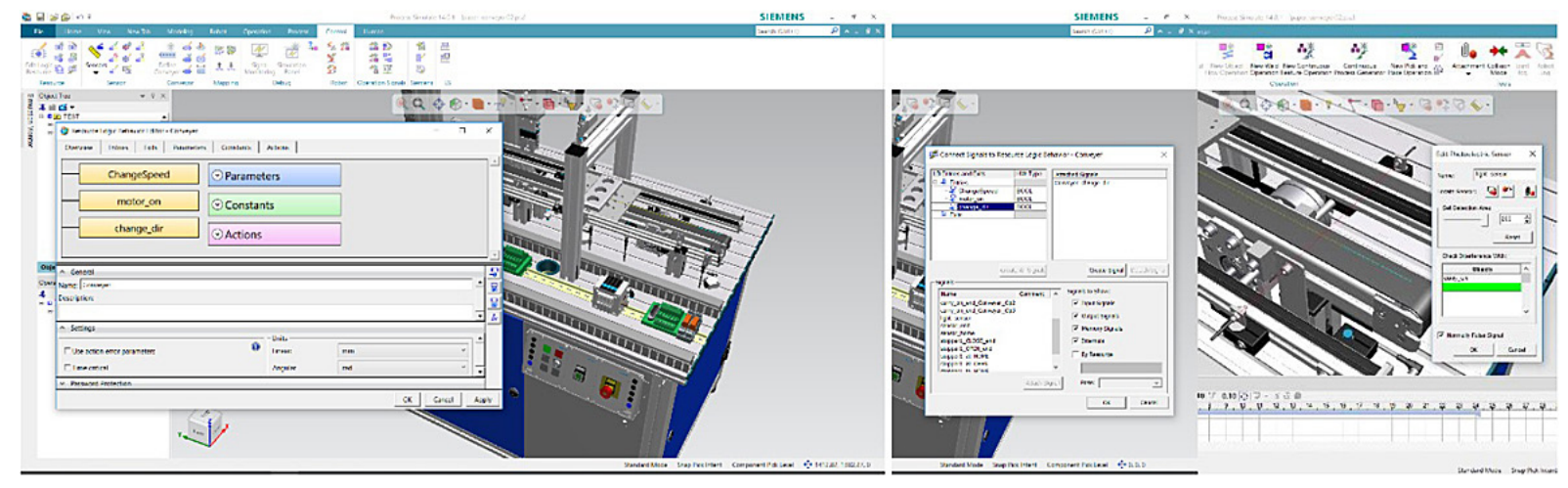

Fig. 6. Definition of the behavior model, parameters with connected signals and editing of the photoelectric sensor

test how kinematically, even in virtual reality. By testing this, it is possible to realistically check the virtual model for whether it corresponds to real reality. It is also possible to check in detail in 3D view, whether there are collisions between the moving parts and using virtual reality to check the individual stop points with certain accuracy [15, 16]. The HTC Vive Headset was selected for virtual reality. In Figure 5 is a preview of the Virtual Reality Tecnomatix Process Simulate module.

\section{Design of the behavior model of the conveyor and sensors integration}

In order to integrate the control system into the virtual conveyor model and thus test the model itself via the PLC controller, several steps need to be taken. One condition is to create a conveyor model behavior. The behavior model is designed to simulate the logical and timebased behavior of real resources compared to the connected control units and therefore consists primarily of the individual simulation components of the devices used in the real device. In Fig. 6 on the left is a model of logic behavior with defined parameters (ChangeSpeed, motor on, change dir). The conveyor control entities (digital inputs and outputs) are also defined, Fig. 6 in the centre. The digital outputs are designed to control the DC motor and move the conveyor away from the manipulator and reverse. A very important factor is the definition of sensors for pallet position detection (inductive sensors) and the definition of the presence of the workpiece through the gate (Set Detection Area, Interference with). In Figure 6 on the right is a red light beam that simulates the infrared beam of light from the optical gate.

\section{Integration of the control system into a virtual model}

The actual virtual commissioning is done by connecting the simulation model with all the attributes mentioned in the previous points and the PLC (virtual or real) control system via standardized interfaces (OPC, Emulator, Bus). If a connection has been made, it is possible to test this connection through the "Sequence Editor". This editor allows you to test individual operations in time or event simulation. The simulation SIMATIC S7-PLCSIM simulator was used to initially test simulation and PLC connection. The program was created via the TIA Portal V14, where the KTP600 HMI panel simulator was connected. The connection of the virtual conveyor model and the TIA portal has been successful, all digital inputs and outputs have been defined with addresses that must be the same whether in the virtual model or in the control program via TIA Portal. In Fig. shows the virtual conveyor model along with the HMI simulator in the operating mode and the test signals on the Simulation Panel, where the green colour is a logical 1 and a red logic 0 . These individual signals can also be activated / forced directly through the Simulation Panel, Figure 7.

\section{Testing the control program on a virtual model}

After verification of the conveyor simulation model as well as virtual reality and achieving integration between the model and the control system, it is possible to test various programming scenarios and optimize the PLC control code directly on the virtual model. The virtual conveyor model behaves conventionally against the control program. In addition, a HMI panel 


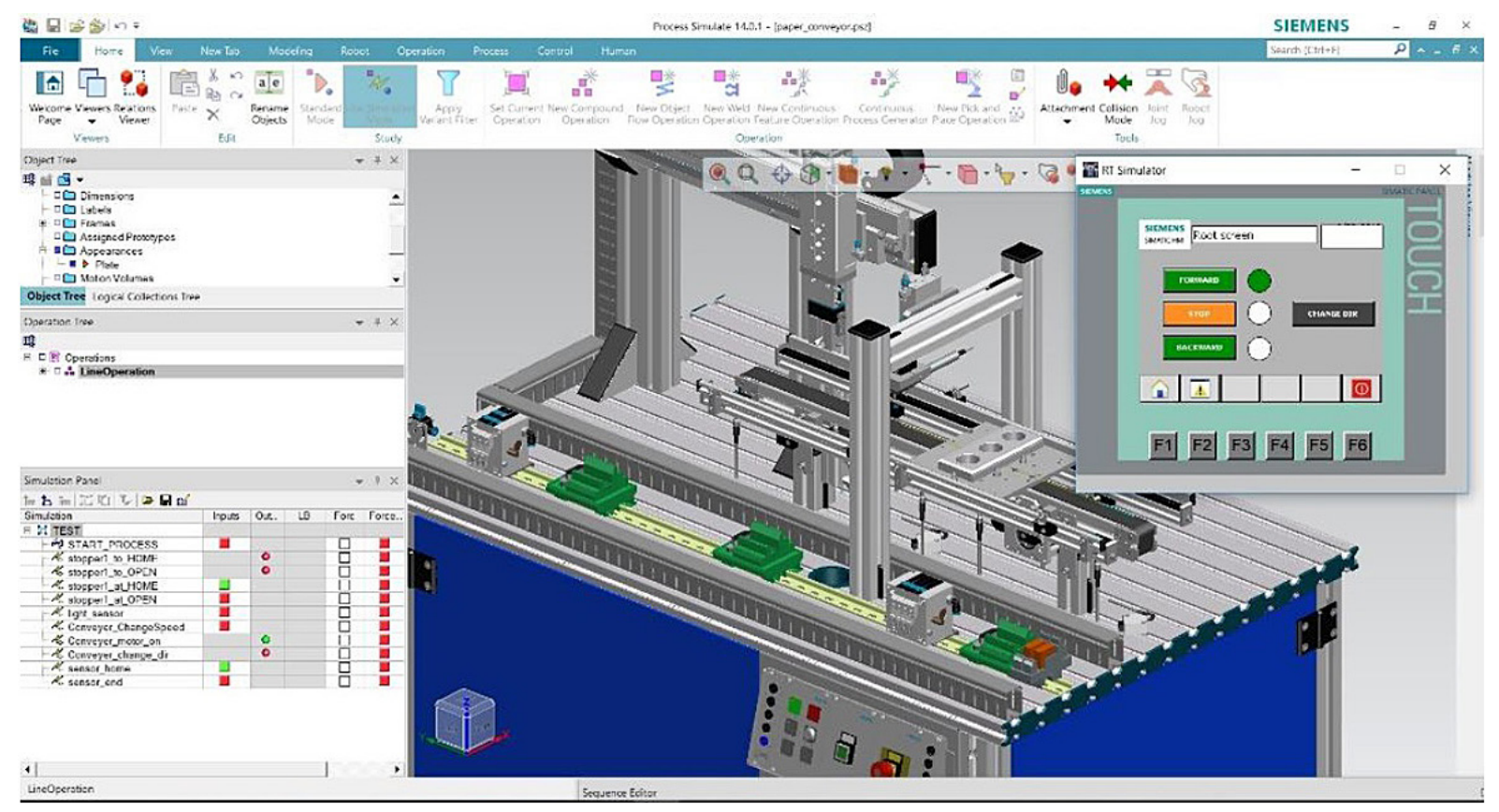

Fig. 7. Definition of the behavior model and parameters with connected signal

with basic signals for manual forward and reverse conveying of the conveyor was also tested, as can also be seen in Figure 8.

\section{DISCUSSION}

The optimization of the control program has been performed with respect to the virtual model created according to material flow requirements. This created control program was then implemented into the actual PLC S7-300 control system on a real conveyor station. The PLC code was generally fine and validated, the differences were only in timer settings. In the future, it would be necessary to connect the real control system directly to the virtual conveyor model and test real model management to get closer to real PLC opti-

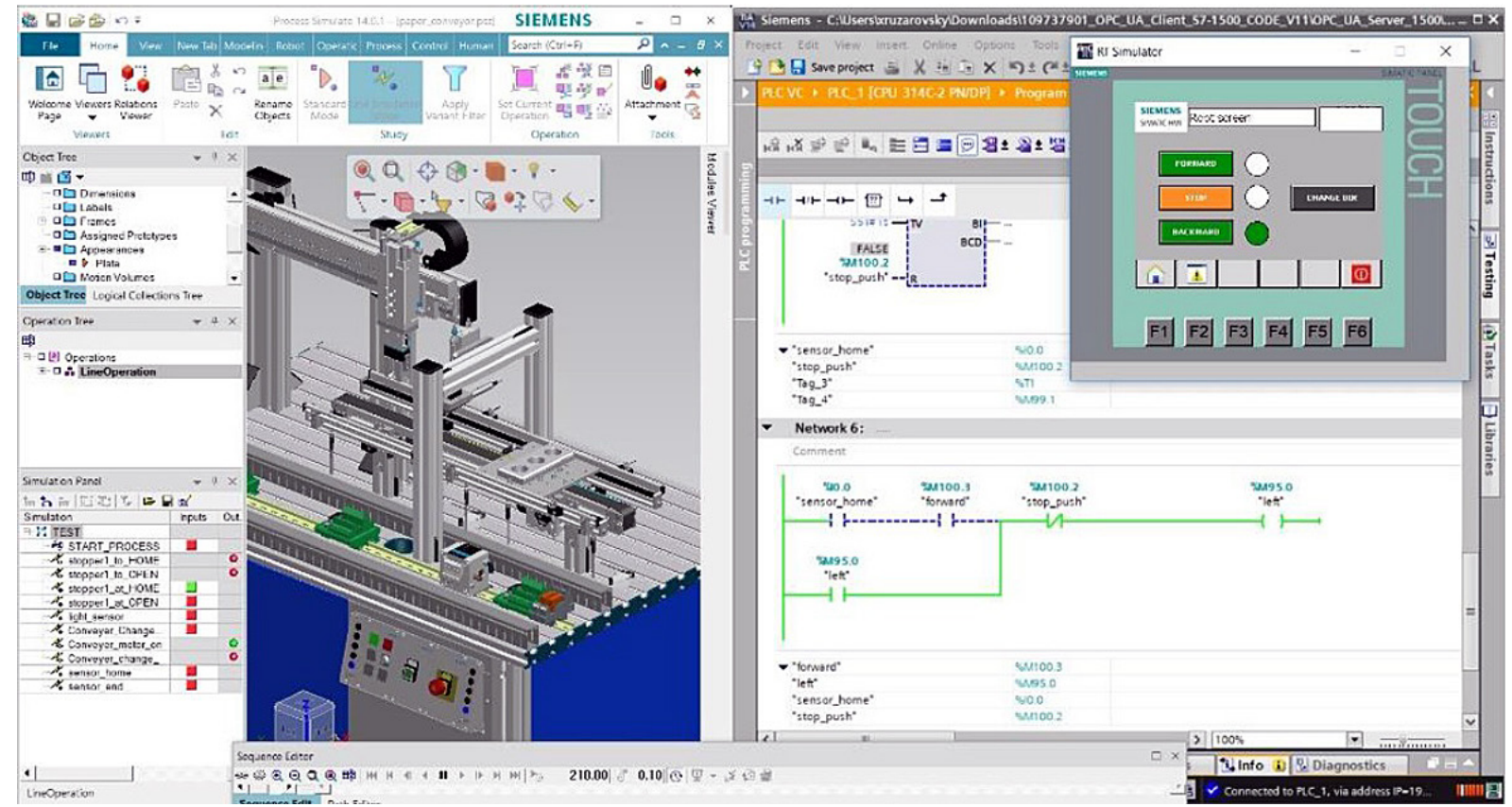

Fig. 8. Verification and validation of the PLC code on the conveyor model through TIA portal and HMI 
mization. A very important factor is also the PLC operating cycle and real-time communication, which the PLC simulation model does not allow.

Overall, virtual commissioning is a very interesting way to save time in developing new conveyors in view of the development of the control program for the control system. The benefits achieved by the simulation application should be greater than the costs needed to implement the simulation and system improvements. The decisive criterion that simulation can be used in practice will be the benefit of its use. The benefits can be divided into quantitative and qualitative. When deciding on a simulation project, a simple rule will apply: Simulation will be warranted if the direct simulation benefits are greater than simulation costs. In many cases, the simulation objective is not a direct economic effect. A supplier of production or transport systems can, for example, demonstrate through simulation their functionality, performance or reliability, which may improve its market position. In order to maximize the effect of simulation, the time at which the simulation is realized varies considerably. The simulation should be used in the early stages of the project, as the greatest potential for improving the system's parameters can be achieved in the early stages, and the associated costs are the lowest in these phases. There are only a few degrees of freedom for change and improvement in the process of implementation and operation, and most of these improvements are associated with high additional costs that often can outweigh the benefits of improvement. This implies that correct and timely decisions have inherently higher benefits than optimization in subsequent stages of the project.

\section{CONCLUSION}

Simulation alone cannot give you an optimal solution, it only allows you to examine the impact of the decision on the simulation model and gives you the choice of the best of them. Virtual commissioning based on verified research has its own perspective because it allows you to test and verify the PLC code on a virtual model that takes place in a traditional design only when an automated device is built and any hardware changes cost money and time. Virtual commissioning requires a realistic digital twin and spends a lot of time with kinematics, behavioral modelling, and signal generation overall. However, when we au- tomate some operations, it is a technology that has great potential and future in designing automated devices.

\section{Acknowledgements}

This paper has been supported by the project KEGA-021STU-4/2018. Development of a laboratory for the design and maintenance of production systems supported by the use of virtual reality. This support is gratefully acknowledged.

\section{REFERENCES}

1. Dahl M., Bengtsson K., Bergagard P., Fabian M., Falkman P. Integrated Virtual Preparation and Commissioning: supporting format methods during automation systems development. IFAC-PapersOnLine 49(12), 2016, 1939-1944.

2. Bergert M. and Kiefer J. Mechatronic Data Models in Production Engineering. IFAC Proceedings Volumes 43 (4), 2010, 60-65.

3. Molnár V., Fedorko G., Andrejiová M., Grinčová A., Michalik P. Online moniroring of a pipe conveyor. Part I: Measurement and analysis of selected operational parameters, Meas. J. Int. Meas. Confed. 94 (2016) 364-371 https://www.sciencedirect. com/science/article/pii/S0263224116304791

4. Reinhart G. and Wünsch G. Economic application of virtual commissioning to mechatronic. Production Engineering - Research and Development 1(4), 2007, 371-379.

5. Fedorko G., Liptai P., Molnár V. Proposal of the methodology for noise sources identification and analysis of continuous transport systems using an acoustic camera. Eng Fail Anal., 83, 2018, 30-46.

6. Seidel S., Donath U. and Haufe J. Towards an integrated simulation and virtual commissioning environment for controls of material handling systems. Proceedings of the 2012 Winter Simulation Conference, 2012.

7. Zhang S., Xia X. Modelling and energy efficiency optimization of belt conveyors. Applied Energy 88(9), 2011, 3061-3071.

8. Puntel-Schmidt P. and Fay A. Levels of Detail and Appropriate Model Types for Virtual Commissioning in Manufacturing Engineering, IFAC-PapersOnLine 48(1), 2015, 922-927.

9. Holubek R. Using Virtual Reality tools to support simulations of manufacturing instances in Process Simulate: The case of an iCIM 3000 system. MATEC Web of Conferences 137, 2017.

10. Vermaak H. and Niemann J. Virtual commissioning: A tool to ensure effective system integration. 
IEEE International Workshop of Electronics, Control, Measurement, Signals and their Application to Mechatronics (ECMSM), 2017.

11. Popovič R., Trebuňa P. and Kliment M. Basic overview about digital factory and virtual commissioning. Acta Logistica - International Scientific Journal about Logistics 2(1), 2015, 1-4.

12. Hossain M. and Semere D.T. Virtual Control System Development Platform with the Application of PLC Device. Proceedings of the International MultiConference of Engineers and Computer Scientists 2013 Vol II, IMECS 2013, March 13 - 15, 2013, Hong Kong, 2013.

13. Lonkwic P., Rozylo P. and Debski H. Numerical and experimental analysis of the progressive gear body with the use of finite-element method. Eksploatacja i Niezawodnosc - Maintenance and Reliability 17(4), 2015, 544-550.

14. Guerrero L.V. Virtual Commissioning with Process Simulation (Tecnomatix). Journal Computer-Aided Design and Applications 11, 2014.

15. Hincapié. Mixing real and virtual components in automated manufacturing systems using PLM tools. International Journal on Interactive Design and Manufacturing 8, 2014, 209-230.

16. Lorenz M. CAD to VR - a methodology for the automated conversion of kinematic CAD models to virtual reality. Research and Innovation in Manufacturing: Proceedings of the 48th CIRP Conference on Manufacturing Systems, 41, 2016. 358-363. 